\title{
Is there a role for pharmacogenetics in the treatment of anorexia nervosa?
}

\author{
"Anorexia nervosa also has one of the highest mortality rates among \\ mental illnesses..."
}

First draft submitted: 22 June 2016; Accepted for publication: 22 June 2016; Published online: 1 August 2016

Keywords: anorexia nervosa $\bullet$ eating disorder $\bullet$ pharmacogenomics

\begin{abstract}
Anorexia nervosa is an eating disorder characterized by restricting dietary intake to the point of low body weight, an intense fear of gaining weight, over-evaluation of shape in one's self appraisal and a disturbance in the way one's body is experienced. DSM-V recognized two subtypes of anorexia nervosa - a restricting subtype which is characterized by only limiting dietary intake, and a binge eating/purging subtype which is characterized by eating large amounts of food and

ties, cardiac problems and decreased bone density [8]. The treatment of anorexia nervosa focuses on restoring body weight, managing physical complications, establishing healthy eating patterns, improving of body image and self-esteem and treating comorbid psychological symptoms [9]. This treatment often consists several stages and intervention types delivered by physicians and allied health providers $[4,9]$ in both inpatient and outpatient settings [10].
\end{abstract} purging them through means such as vomiting, laxatives or excessive exercise [1]. Prior definitions of anorexia nervosa included criteria that affected patients weigh less than $85 \%$ of expected weight for their age and height and that post-menarcheal females stop menstruating [2], but these requirements have been removed from DSM-V to better reflect the diversity of this illness.

The prevalence of anorexia nervosa is reported to be between 0.2 and $0.9 \%$ of the population $[3,4]$ with women comprising the majority of patients diagnosed [5]. The course and outcomes of anorexia nervosa are highly variable with only half of patients ever attaining recovery and a quarter to fifth remaining seriously, chronically ill $[5,6]$. Anorexia nervosa also has one of the highest mortality rates among mental illnesses [7] with 5-15\% of those affected dying of the illness [5,6]. Psychiatric complications include social impairment, professional impairment, mood disorders, severe anxiety, substance abuse and suicide [4]. Medical complications include gastric dysfunction, electrolyte abnormali-

\section{But what causes anorexia nervosa?}

The etiology of anorexia nervosa appears to be multifactorial with genetic, neurobiological and psychological factors contributing to its development and maintenance [10,11]. Proposed contributing factors include serotonergic dysfunction [12], dopamingergic dysfunction [12] and disturbances in the metabolism of norepinephrine [13]. Genetic research in eating disorders patients has found associations between candidate genes [14], alleles [15] and polymorphic variants [16], but these have not changed our understanding of etiology. Indeed, what causes anorexia nervosa remains not fully understood [11].

Proposed pharmacological treatment for anorexia nervosa have been based on these biological theories, neurobiological research into the control of appetite and food intake, on clinical observations and uncontrolled studies [9]. Specifically, medications have been trialed to target maladaptive thought patterns, stimulate appetite, treat comorbid symptoms and prevent relapse after recov-
Sarah Smith

Program for Eating Disorders, Department of Psychiatry, Toronto General Hospital, Toronto, Canada

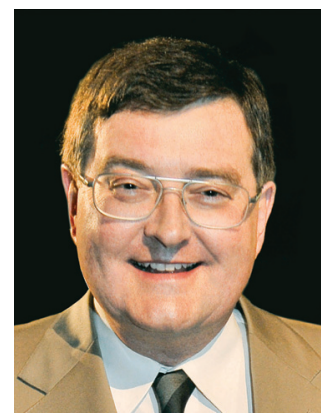

Blake Woodside

Author for correspondence:

Program for Eating Disorders,

Department of Psychiatry, Toronto General Hospital, Toronto, Canada

Tel.: +1 4163404445

Fax: +1 4163404198

b.woodside@utoronto.ca 
ery [17]. Yet the results of this research have been largely disappointing $[10,18]$.

Indeed, to date there is no established or recommended pharmacologic treatment for anorexia nervosa [18,19].

Prior research has explored the potential applications of antidepressants, antipsychotics, nutritional supplements and hormonal treatments with mixed results $[9,17,18,20]$. Historically, tricyclic antidepressants initially appeared promising but later randomized control trials did not support their routine use $[10,20]$. As their use has also been associated with fatal cardiac arrthymias in patients with low body weight they are no longer recommended for clinical use in anorexia nervosa $[10,20]$. Similarly, lithium was reported to have benefit in weight restoration in early research but the electrolyte changes associated with starvation make its' use medically dangerous $[10,20]$. Early research on selective serotonin research uptake inhibitors also appeared promising but larger studies with longer follow-up reported no benefit in weight stabilization or relapse prevention $[10,20]$.

Most recently, attention has turned to a potential role for antipsychotic medications in the treatment of anorexia nervosa. This work was predicated on an interpretation as anorexia nervosa patient's perceptions of their bodies as delusional. Second generation antipsychotics in particular were proposed as a way to treat serotonergic and dopaminergic dysfunction, to manage comorbid anxiety and depression and to induce weight gain [21]. Initial work on this hypothesis again seemed promising with several small studies reporting positive weight gain and decreased eating disorder psychopathology $[10,20]$. However, most of these studies had small sample sizes, unblended participants and short periods of follow-up [22]. These studies have also been included in a two recent meta-analyses of randomized control trials of antipsychotics in anorexia nervosa that have reported no significant benefits in weight gain [9,21], eating disorder attitudes, anxiety or depressive symptoms in anorexia nervosa with second-generation antipsychotics when compared with placebo [21]. The exception to this conclusion was very limited data suggesting that quetiapine can decrease comorbid anxiety and improve eating disorder attitudes [21] based on one study that has not been replicated. Importantly, second-generation antipsychotic side effects including akathesia, drowsiness and elevated blood sugar have

\section{References}

1 American Psychiatric Association. Diagnostic and statistical manual of mental disorders: DSM-5. American Psychiatric Association, Washington, DC, USA (2013).

2 American Psychiatric Association. Diagnostic and statistical manual of mental disorders: DSM-IV. American Psychiatric Association, Washington, DC, USA (1994). also reported been reported in several studies [21], further calling the potential balance between benefit and risk for into question.

\section{Is there then a role for pharmacogenetics in the treatment of anorexia nervosa?}

Based on the lack of evidence for pharmacological treatment of anorexia nervosa the answer to this question is not clear.

Clinically, as in the absence recommended pharmacologic treatment for anorexia nervosa $[18,19]$ there does not yet appear to be a role for pharmacogenetics in the treatment of the primary disorder.

However, as it has been reported, even among negative trials, that some participants benefit from pharmacotherapy it has been suggested that medications may have benefit for subsets of anorexia nervosa patients [22]. Specifically, it has been proposed that these patients benefit from the treatment of co-morbid anxiety, obsessionality and cognitive rigidity $[18,22]$. Patients with anorexia nervosa are known to have high rates of anxiety and mood disorders [4]. It is possible that as the field of pharmacogenetic research on antidepressant and anti-anxiety medications progresses that this work could influence how we treat these co-morbid disorders in patients with anorexia nervosa. It is also possible that ongoing genetic research into the etiology of anorexia nervosa could yield clinically useful information about etiology and by extension clinical treatment. However, to date there remains a disconnection between the size of eating disorders as a public health problem and amount of research being done on their pharmacological treatment [22]. Additional research is needed to better understand the genetics, neurobiology and course of anorexia nervosa before we will know whether there is a role for pharmacogenetics in its' treatment.

\section{Financial \& competing interests disclosure}

The authors have no relevant affiliations or financial involvement with any organization or entity with a financial interest in or financial conflict with the subject matter or materials discussed in the manuscript. This includes employment, consultancies, honoraria, stock ownership or options, expert testimony, grants or patents received or pending, or royalties.

No writing assistance was utilized in the production of this manuscript.

3 Hoek HW, Van Hoeken D. Review of the prevalence and incidence of eating disorders. Int. J. Eat. Disord. 34(4), 383-396 (2003).

4 Hudson JI, Hiripi E, Pope HG, Kessler RC. The prevalence and correlates of eating disorders in the national comorbidity survey replication. Biol. Psychiatry 61(3), 348-358 (2007). 
5 Steinhausen H.C. The outcome of anorexia nervosa in the 20th century. Am. J. Psychiatry 159(8), 1284-1293 (2002).

6 Zipfel S, Löwe B, Reas DL, Deter HC, Herzog D. Long-term prognosis in anorexia nervosa lessons from a 21-year followup study. Lancet 355(9205), 721-722 (2000).

7 Harris EC, Barraclough B. Excess mortality of mental disorder. Br. J. Psychiatry 173, 11-53 (1998).

8 Mitchell JE, Crow S. Medical complications of anorexia nervosa and bulimia nervosa. Curr. Opin. Psychiatry 19(4), 438-443 (2006).

9 de Vos J, Houtzager L, Katsaragaki G, van de Berg E, Cuijpers P, Dekker J. Meta analysis on the efficacy of pharmacotherapy versus placebo on anorexia nervosa. J. Eat. Disord. 2(1), 27 (2014).

10 Miniati M, Mauri M, Ciberti A, Mariani MG, Marazzitti D, Dell'Osso L. Psychopharmacological options for adult patients with anorexia nervosa. CNS Spectr. 21(2), 134-142 (2016).

11 Shiha PB, Woodside DB. Contemporary views on the genetics of anorexia nervosa. Eur. Neuropsychopharmacol. 26(4), 663-673 (2016).

12 Bosanac P, Norman T, Burrows G, Beaumont P. Serotonergic and dopaminergic systems in anorexia nervosa: a role for atypical antipsychotics? Aust. NZ J. Psychiatry 39(3), 146-153 (2005).

13 Pirke KM. Central and peripheral noradrenaline regulation in eating disorders. Psych. Res. 62, 43-49 (1996).

14 Adan RA, Vink T. Drug target discovery by pharmacogenetics: mutations in the melanocortin system and eating disorders. Eur. Neuropsychopharmacol. 11(6), 483-490 (2001).

15 Gorwood P. Eating disorders, serotonin transporter polymorphisms and potential treatment response. Am. J. Pharmacogenomics 4(1), 9-17 (2004).

16 Monteleone P, Maj M. Genetic susceptibility to eating disorders: associated polymorphisms and pharmacogenetic suggestions. Pharmacogenomics 9(10), 1487-1520 (2008).

17 Hebebrand J. Pharmacotherapy of anorexia nervosa: more questions than answers. J. Am. Acad. Child Adolesc. Psychiatry 50(9), 854-856 (2011).

18 Crow SJ, Mitchell JE, Roerig JD, Steffen K. what potential role is there for medication treatment in anorexia nervosa? Int. J. Eat. Disord. 42(1), 1-8 (2009).

19 Agras WS, Robinson AH. Forty years of progress in the treatment of the eating disorders. Nord. J. Psychiatry 62(Suppl. 47), 19-24 (2008).

20 Flament MF, Bissada H, Spettigue W. Evidence-based pharmacotherapy of eating disorders. Int. J. Neuropsychopharmacol. 15(2), 189-207 (2012).

21 Kishi T, Kafantaris V, Sunday S, Sheridan EM, Correll CU. Are antipsychotics effective for the treatment of anorexia nervosa? Results from a systematic review and meta-analysis. J. Clin. Psychiatry 73, 757-766 (2012).

22 McElroy SL, Guerdjikova AI, Mori N, Keck PE Jr. Psychopharmacologic treatment of eating disorders: emerging findings. Curr. Psychiatry Rep. 17(5), 35 (2015). 